\title{
Microrganismos e Resistência Antimicrobiana em feridas complexas
}

\author{
Microorganisms and Antimicrobial Resistance in complex wounds \\ Microorganismos y Resistencia Antimicrobiana en heridas complejas
}

Recebido: 19/01/2021 | Revisado: 23/01/2021 | Aceito: 29/01/2021 | Publicado: 06/02/2021

Fabianny Torres de Oliveira

ORCID: https://orcid.org/0000-0001-6193-2002

Universidade Federal de Alagoas, Brasil

E-mail: fabianny_torres@ hotmail.com.br

Gabriella Keren Silva Lima

ORCID: https://orcid.org/0000-0001-6912-5985 Instituto de Medicina Integral Professor Fernando Figueira, Brasil E-mail: gabriellaklima@hotmail.com.br

Igor Michel Ramos dos Santos

ORCID: https://orcid.org/0000-0001-6557-3369 Universidade Federal de Alagoas, Brasil E-mail: igor.santos@esenfar.ufal.br

Davi Porfirio da Silva

ORCID: httpS://orcid.org/0000-0002-1856-4512 Universidade Federal de Alagoas, Brasil

E-mail: daviporfirio14@hotmail.com

Thaís Honorio Lins Bernardo

ORCID: https://orcid.org/0000-0002-8058-8400

Universidade Federal de Alagoas, Brasil

E-mail: thais.bernardo@esenfar.ufal.br

Maria Lysete de Assis Bastos

ORCID: https://orcid.org/0000-0003-1752-7645

Instituto Federal de Alagoas, Brasil

E-mail: lysetebastos@gmail.com

Paulo Sérgio Gomes da Silva

ORCID: https://orcid.org/0000-0002-5720-6638

Universidade Federal de Alagoas, Brasil

E-mail: enfermagemheha@gmail.com

Denise de Almeida Barros

ORCID: https://orcid.org/0000-0002-7806-3656

Secretaria Municipal de Saúde de Estrela de Alagoas, Brasil

E-mail: denisebarros.enf@hotmail.com

\begin{abstract}
Resumo
Objetivo: Caracterizar a microbiota de feridas complexas e a resistência antimicrobiana dos microrganismos encontrados. Métodos: Estudo transversal de cunho observacional e dissertativo, realizado a partir dos resultados de culturas microbiológicas de pacientes em acompanhamento ambulatorial para tratamento de feridas complexas. Estudo aprovado sob parecer CAAE número 93044418.6.0000.5013. Resultados: Foram analisados 110 exames, coletados a partir de lesões do tipo vasculogênica, diabética, lesão por pressão, cirúrgica, oncológica, aguda, antraz, síndrome de fournier e úlcera de marjolin. Foram identificadas 22 espécies de bactérias, sendo 18 Gram-negativas e 4 Gram-positivas, predominando as espécies Pseudomonas aeruginosas, Proteus mirabilis e Staphylococcus aureus. Todos os microrganismos identificados apresentaram resistências a, pelo menos, um antimicrobiano, sendo a espécie P. mirabilis com resistência a um maior número de antibióticos. Conclusão: Esse estudo aponta que a microbiota de feridas complexas é predominantemente composta por microrganismos Gram-negativos, destaca-se a espécie $P$. aeruginosa. A resistência antimicrobiana foi detectada em todos os microrganismos encontrados.
\end{abstract}

Palavras-chave: Ferimentos e lesões; Microbiota; Infecção dos ferimentos; Resistência microbiana a medicamentos.

\begin{abstract}
Objective: To characterize the microbiota of complex wounds and the antimicrobial resistance of the microorganisms found. Methods: Cross-sectional, observational and dissertative study, carried out based on the results of microbiological cultures of patients undergoing outpatient follow-up to treat complex wounds. Study approved under CAAE opinion number 93044418.6.0000.5013. Results: 110 tests were analyzed, collected from vasculogenic, diabetic, pressure ulcer, surgical, oncological, acute, anthrax lesions, fournier syndrome and marjolin ulcer. 22 species of bacteria were identified, 18 Gram-negative and 4 Gram-positive, with predominance of Pseudomonas aeruginosas, Proteus mirabilis and Staphylococcus aureus. All the microorganisms identified showed resistance to at least one antimicrobial, the $P$. mirabilis species being resistant to a greater number of antibiotics. Conclusion: This study points
\end{abstract}


out that the complex wound microbiota is predominantly composed of Gram-negative microorganisms, the species $P$. aeruginosa stands out. Antimicrobial resistance was detected in all microorganisms found.

Keywords: Wounds and injuries; Microbiota; Wound infection; Drug resistance microbial.

\section{Resumen}

Objetivo: Caracterizar la microbiota de heridas complejas y la resistencia antimicrobiana de los microorganismos encontrados. Métodos: estudio de corte transversal, observacional y tesis, realizado a partir de los resultados de cultivos microbiológicos de pacientes sometidos a seguimiento ambulatorio para el tratamiento de heridas complejas. Estudio aprobado bajo opinión CAAE número 93044418.6.0000.5013. Resultados: Se analizaron 110 pruebas, recogidas de lesiones vasculogénicas, diabéticas, de presión, quirúrgicas, oncológicas, agudas, ántrax, síndrome de Fournier y úlcera de marjolina. Se identificaron 22 especies de bacterias, 18 Gram negativas y 4 Gram positivas, con predominio de Pseudomonas aeruginosas, Proteus mirabilis y Staphylococcus aureus. Todos los microorganismos identificados mostraron resistencia a al menos un antimicrobiano, siendo la especie $P$. mirabilis resistente a un mayor número de antibióticos. Conclusión: Este estudio señala que la microbiota compleja de la herida está compuesta predominantemente por microorganismos Gram-negativos, se destaca la especie $P$. aeruginosa. Se detectó resistencia a los antimicrobianos en todos los microorganismos encontrados.

Palabras clave: Heridas y traumatismos; Microbiota; Infección de heridas; Farmacorresistencia microbiana.

\section{Introdução}

As feridas são consideradas rupturas estruturais ou fisiológicas da pele que provocam respostas de reparação baseadas na interação de células inflamatórias e seus mediadores. Para avaliação dessas rupturas, com vistas ao fechamento da lesão, deve-se levar em consideração critérios clínicos pertinentes ao processo cicatricial, dentre eles, o que diz respeito ao tempo de aparecimento da lesão. Nesse sentido, as feridas são classificadas em agudas ou crônicas. Essas são assim denominadas em virtude do processo de cicatrização durar mais de doze semanas. Essas lesões podem ser responsáveis por índices de morbimortalidade significativos, além de um impacto econômico considerável. Como na maioria dos casos ocorre a presença de infecção na lesão, então os maiores gastos estão relacionados ao uso de agentes antimicrobianos (Pires, 2018).

$\mathrm{O}$ aumento de citocinas pro-inflamatórias e os fatores predisponentes do portador podem resultar na cronicidade da lesão, favorecendo a colonização microbiana. No entanto, o sistema imune atua realizando o controle da carga microbiana local, auxiliando assim na cicatrização da lesão, mesmo que de forma lenta. O comprometimento do sistema imunológico pode ocasionar um aumento desenfreado da carga microbiana resultando em colonização crítica, infecção local ou sistêmica (Tedeschi, et al., 2017).

Frente ao apresentado, este estudo tem como objeto de pesquisa, a microbiota bacteriana e a resistência antimicrobiana identificada em lesões complexas de pacientes acompanhados ambulatoriamente em um serviço de referência para o tratamento de feridas. Visto que, caracterizar os microrganismos, bem como a suscetibilidade e resistência bacteriana em serviços de saúde é imprescindível, para se entender a complexidade do portador de lesão sob os aspectos: ferida, custo de tratamento, impacto social e, mais ainda, a relação atual crescente entre microbiota, uso indiscriminado de antibióticos e resistência bacteriana.

Para a avaliação da carga microbiana de um tecido e diagnóstico de infecção em feridas realiza-se a coleta no leito da lesão para cultura, dentre três técnicas possíveis: biópsia, swab ou aspirado (Haalboom, et al., 2019). A epidemiologia microbiológica determinará a descrição dos microrganismos presentes segundo a etiologia, sensibilidade e resistência antimicrobiana. O conhecimento microbiológico da lesão é determinante para escolha das condutas de tratamento, local ou sistêmico (Tedeschi, et al., 2017).

Desta forma, conhecer o perfil microbiológico do serviço de saúde no qual se atua torna-se uma ferramenta útil, com sustentação à luz da ciência e subsidia o profissional na tomada de decisão clínica em situações indispensáveis à adoção de antibioticoterapia empírica e de terapia antimicrobiana tópica.

Esse estudo foi desenvolvido a fim de responder à questão norteadora: Quais as bactérias mais prevalentes e a 
resistência antimicrobiana nas lesões dos pacientes atendidos em um serviço de referência para o tratamento de feridas? Desse modo, o objetivo foi caracterizar a microbiota de feridas complexas e a resistência antimicrobiana dos microrganismos encontrados.

\section{Metodologia}

Trata-se de um estudo quantitativo transversal, de cunho observacional e dissertativo (Rodrigues, et al., 2017), que se utilizou de dados institucionais referentes aos resultados de culturas microbiológicas de feridas, em pacientes acompanhados em um Ambulatório de Feridas Complexas e de Estomias, numa instituição pública de ensino do nordeste brasileiro, no período de setembro de 2018 a setembro de 2019, correspondendo ao primeiro ano de implantação do serviço.

Foram incluídos na pesquisa dados secundários, coletados no Prontuário Eletrônico do Paciente (PEP), de todos os indivíduos acompanhados no ambulatório de feridas da instituição que, por decisão clínica da equipe, realizou cultura de ferida no período estabelecido. Foram excluídos os admitidos fora dos conformes temporais estabelecidos, menores de 18 anos, pacientes estomizados e os resultados sugestivos de contaminação. Desse modo, a amostra final foi composta de 110 pacientes.

Os exames de cultura foram realizados pelos enfermeiros da equipe, mediante identificação clínica de colonização crítica, infecção ou mediante solicitação médica. Para coleta foi realizada limpeza do leito da lesão com soro fisiológico a $0,9 \%$ e desbridamento instrumental conservador, na presença de tecidos desvitalizados, a fim de viabilizar a região da coleta; coleta com técnica estéril por Swab de $150 \mathrm{~mm}$ de comprimento e ponta de Rayon em meio Stuart; envio de imediato ao Laboratório de Análises Clínicas da Instituição para análise, conforme protocolo laboratorial da instituição, por método de Gram em sistema automatizado do tipo BD Phoenix.

Os dados colhidos no PEP se referem a sexo, idade, tipo de lesão, resultado da cultura de ferida, espécie identificada e resultado do antibiograma, que foram tabulados em uma planilha do Microsoft Office Excel® 2016 e realizada análise estatística descritiva.

O projeto de pesquisa foi apreciado pelo comitê de ética em pesquisa e aprovado sob parecer CAAE número 93044418.6.0000.5013. Os aspectos éticos foram respeitados em todo momento, tanto na observância de critérios para coleta confiável dos dados, quanto para garantia do sigilo na identificação do paciente, obedecendo as resoluções no 466/2012 e nº 510/2016 do Conselho Nacional de Saúde do Ministério da Saúde.

\section{Resultados}

Dentre os 110 pacientes submetidos à coleta para análise microbiológica, 56 (50,9\%) foram do sexo feminino e 54 $(49,1 \%)$ do masculino. Sendo, $2(1,8 \%)$ com idade maior ou igual a 18 anos, $57(51,8 \%)$ na faixa etária entre 20 a 59 anos e 51 $(46,4 \%)$ com 60 anos ou mais.

Em $64(58,1 \%)$ lesões, apenas um tipo de microrganismo foi encontrado, enquanto em 13 (11,8\%) dos resultados foram encontrados dois tipos de microrganismos, $4(3,6 \%)$ apareceram três distintas espécies e em apenas $1(0,9 \%)$ das lesões foram encontrados quatro microrganismos diferentes.

Foram identificadas 187 culturas de feridas realizadas no período estabelecido pelo estudo. Entretanto, identificou-se, que haviam pacientes com mais de uma coleta, sendo 124 positivas para a presença de microrganismos. As culturas foram coletadas de 110 lesões: 56 (50,9\%) vasculogênicas, 23 (21\%) diabéticas, 10 (9,1\%) cirúrgicas, 4 (3,6\%) oncológicas, 4 (3,6\%) agudas, $1(0,9 \%)$ antraz, $1(0,9 \%)$ úlcera de Marjolin e $1(0,9 \%)$ Síndrome de Fournier.

Em relação as 124 amostras positivas, foram identificados 22 microrganismos. Destes 18 eram bactérias Gramnegativas: Pseudomonas aeruginosa 47 (38\%), Proteus mirabilis 16 (12,9\%), Klebsiella pneumoniae 7 (5,6\%), Escherichia Coli 6 (4,8\%), Providencia stuartii 6 (4,8\%), Enterobacter clocae 5 (4\%), Acinetobacter baumanii 4 (3,3\%), Enterobacter 
aerogenes 4 (3,3\%), Morganella morganii 2 (1,6\%), Serratia. marcescens 3 (2,4\%), Proteus vulgaris 2 (1,6\%), Providencia rettgeri 2 (1,6\%), Acinetobacter baumanii/calcoaceticus complexo 1 (0,8\%), Burkholderia cepacia 1 (0,8\%), Citrobacter koseri 1 (0,8\%), Citrobacter spp 1 (0,8\%), Pantoea agglomerans 1 (0,8\%), Providencia alcalifaciens 1 (0,8\%).

Dentre as bactérias Gram-positivas foram identificados 4 tipos: Staphylococcus. aureus 12 (9,7\%), Staphylococcus haemolyticus 1 (0,8\%), Staphylococcus sciuri 1 (0,8\%), Streptococcus agalactiae 1 (0,8\%). Sendo a proporção de Gramnegativa para Gram-positiva de 7:1.

A relação da etiologia das lesões e microrganismos evidencia que as bactérias Gram-negativas estiveram presentes em todos os tipos de lesão, conforme apresentado na Tabela 1, sendo a Gram-negativa P. aeruginosa a que aparece em 6 (38,14\%) tipos das lesões analisadas nesse estudo, sendo a lesão de etiologia vasculogênica a que mais apresentou esse microrganismo.

Tabela 1. Relação entre etiologia da lesão e microrganismos identificados nos exames de cultura de feridas. Maceió, Brasil, 2018 a 2019.

\begin{tabular}{|c|c|c|c|c|c|c|}
\hline & 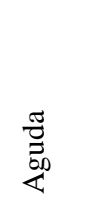 & 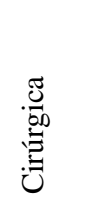 & $\hat{\mathrm{a}}$ & 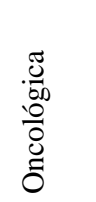 & 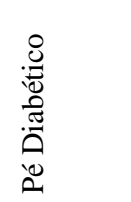 & 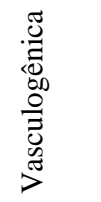 \\
\hline Pseudomonas aeruginosas & $2(1,6)$ & $3(2,4)$ & $1(0,8)$ & $2(1,6)$ & $13(10,7)$ & $26(21)$ \\
\hline Proteus mirabilis & $1(0,8)$ & $4(3,2)$ & $1(0,8)$ & $1(0,8)$ & $1(0,8)$ & $8(6,5)$ \\
\hline Klebsiella pneumoniae & & $1(0,8)$ & & $1(0,8)$ & $2(1,6)$ & $3(2,4)$ \\
\hline Escherichia Coli & & $2(1,6)$ & $1(0,8)$ & & $2(1,6)$ & $1(0,8)$ \\
\hline Providencia stuartii & & & $1(0,8)$ & & & $5(4.1)$ \\
\hline Enterobacter clocae & & $1(0,8)$ & & & & $4(3,2)$ \\
\hline Acinetobacter baumanii & & $1(0,7)$ & & & $3(2,4)$ & \\
\hline Enterobacter aerogenes & & & $1(0,8)$ & & $2(1,6)$ & $1(0,8)$ \\
\hline Morganella morganii & & & & & & $2(1,6)$ \\
\hline Serratia marcescens & & & & & & $3(2,4)$ \\
\hline Proteus vulgaris & & & & & & $1(0,8)$ \\
\hline Providencia rettgeri & & & & & $1(0,8)$ & $1(0,8)$ \\
\hline Acinetobacter baumanii/calcoaceticus complexo & & & & & $1(0,8)$ & \\
\hline Burkholderia cepacia & & & & & & $1(0,8)$ \\
\hline Citrobacter koseri & & & & & & $1(0,8)$ \\
\hline Citrobacter spp & & & & & & $1(0,8)$ \\
\hline Pantoea agglomerans & $1(0,8)$ & & & & & \\
\hline Providencia alcalifaciens & & & & & & $1(0,8)$ \\
\hline Staphylococcus aureus & & & $2(1,6)$ & & $1(0,8)$ & $9(7,3)$ \\
\hline
\end{tabular}


Fonte: Autores (2020).

Na realização do antibiograma, o microrganismo que apresentou resistência a um maior número de antibióticos foi a Gram-negativa P. mirabilis, em 19 (59,3\%) dos medicamentos. Em relação aos antibióticos, a resistência apresentada pelos microrganismos foi mais forte frente à Ampicilina e menos ao Sulfametoxazol associado com Trimetoprima e Amicacina (Tabela 2).

Tabela 2. Relação entre os antibióticos e os principais microrganismos que desenvolveram resistência nos exames de cultura de feridas. Maceió, Brasil, 2018 a 2019.

\begin{tabular}{|c|c|c|c|c|c|c|c|c|}
\hline \multirow[t]{2}{*}{ Antibiótico } & \multicolumn{8}{|c|}{ Resistência N. (\%) } \\
\hline & $E A$ & $E C$ & $E C O$ & $K P$ & $P S$ & $P S T$ & $P A$ & $S A$ \\
\hline Amicacina & & & & & & & $3(23)$ & 1(9) \\
\hline Ampicilina & $4(100)$ & $5(100)$ & $5(83,3)$ & $6(100)$ & $14(100)$ & $6(100)$ & & $5(45,4)$ \\
\hline Ampicilina/ & & & & & & & & \\
\hline sulbactam & $3(75)$ & $3(60)$ & $1(16,6)$ & $1(16,6)$ & $3(21,4)$ & & & \\
\hline Amoxicilina-clavulanato & $1(25)$ & $1(20)$ & $1(16,6)$ & $1(16,6)$ & $6(42,8)$ & $6(100)$ & $2(15,3)$ & \\
\hline Aztreonam & & & & & & & $1(7,6)$ & \\
\hline Cefazolina & $2(50)$ & $3(60)$ & $1(16,6)$ & $2(33,3)$ & $6(42,8)$ & & & \\
\hline Cefepima & & & $1(16,6)$ & $2(33,3)$ & $2(14,2)$ & $1(16,6)$ & $8(61,5)$ & \\
\hline Cefoxitina & $4(100)$ & $5(100)$ & $1(16,6)$ & $2(33,3)$ & $3(21,4)$ & $1(16,6)$ & $2(15,3)$ & \\
\hline Cefuroxima & $1(25)$ & & $1(16,6)$ & $1(16,6)$ & $4(28,5)$ & $2(33,3)$ & & \\
\hline Colistina & & & $1(16,6)$ & & $7(50)$ & & $6(46,1)$ & \\
\hline Ceftriaxona & $1(25)$ & $3(60)$ & $1(16,6)$ & $3(50)$ & $10(71,4)$ & $1(16,6)$ & & \\
\hline Clindamicina & & & & & & & & $8(72,7)$ \\
\hline Ciprofloxacina & $3(75)$ & $1(20)$ & $6(100)$ & $6(100)$ & $8(57,1)$ & $1(16,6)$ & 13(100) & \\
\hline Ertapenem & $1(25)$ & $2(40)$ & $3(50)$ & & $8(57,1)$ & $2(33,3)$ & & \\
\hline Eritromicina & & & & & & & & $9(81,8)$ \\
\hline Gentamicina & & & $2(33,3$ & $3(50)$ & $8(57,1)$ & $6(100)$ & $10(76,9)$ & \\
\hline Imipenem & $1(25)$ & & & $1(16,6)$ & & & $5(38,4)$ & \\
\hline Levofloxacina & $1(25)$ & $1(20)$ & $6(100)$ & $5(83,3)$ & $6(42,8)$ & & $12(92,3)$ & \\
\hline Meropenem & & & $1(50)$ & & $2(14,2)$ & $1(16,6)$ & $2(15,3)$ & \\
\hline Minociclina & & & & & & & & $1(9)$ \\
\hline
\end{tabular}


Penicilina G

Piperaclina/

tazobactam

Rifampicina

Tigeciclina

Tetraciclina

Trimetoprim-sulfametoxazol
2(50)

$1(20)$

2(40)

$2(33,3)$

$5(35,7)$

$11(78,5)$

$4(28,5)$

$3(50)$

$7(50)$

$3(50)$

$2(18,1)$

Legenda: E. aerogenes (EA); E. cloacae $(E C) ;$ E. coli $(E C O) ;$ K. pneumoniae $(K P) ; P$. mirabilis $(P M) ; P$. stuartii $(P S) ; P$. stuartii $(P S T) ; P$. aeruginosa (PA); S. aureus (SA). Fonte: Autores (2020).

\section{Discussão}

Feridas são problemas de saúde pública, trazem impacto ao indivíduo e a sociedade devido à interferência nos aspectos biopsicossociais de seu portador e aos elevados custos aos serviços de saúde causados pelo tratamento. Um dos fatores relacionados à complexidade das feridas é a infecção microbiana, tratamento antimicrobiano e, não obstante, a resistência bacteriana (Santos, et al., 2018).

O crescimento bacteriano é um dos principais pré-requisitos para desenvolvimento de uma infecção, isso inclui o tipo de microrganismo e as toxinas sintetizadas por eles (Santos, et al., 2016). A infecção é uma complicação que retarda a cicatrização, pois pode prolongar a fase inflamatória da cicatrização ao provocar destruição tecidual e retardo da produção de colágeno e dos componentes da epitelização (Campos, et al., 2016).

As feridas podem apresentar mais de um tipo de microrganismo devido à pele estar exposta a microbiota corpórea e ambiental, podendo ocasionar além de contaminação múltipla, infecção por microrganismos oportunistas (Souza, et al., 2016). Nesse sentido, existe a relação de úlceras infectadas por apenas um patógeno com melhor prognóstico, que aquelas com infecção polimicrobiana, que tendem a infecção grave e amputações (Silva, et al., 2018).

Em relação à microbiota por tipo de ferida, as lesões vasculogênicas, conforme a literatura, apresenta como principais bactérias isoladas as espécies $S$. aureus, P. aeruginosa, P. mirabillis, E. aerogenes e E. coli, com destaque para as três primeiras (Borges, Amorim \& Carvalho, 2014). Quando comparado aos resultados deste estudo, os mesmos microrganismos foram isolados, porém com diferença significativa, pois há inversão em relação à prevalência dos dois primeiros, sendo uma bactéria Gram-negativa a maior responsável pela infecção das lesões vasculogênicas neste estudo.

Em pacientes com lesões caracterizadas como pés diabéticos, o grupo de bactérias mais frequente na literatura foi das enterobactérias, seguido por estafilococos (Oliveira \& Oliveira, 2014). Enquanto a referida pesquisa revelou P. Aeruginosa e A. baumanii como mais presentes no leito das lesões de pés diabéticos.

Em lesões cirúrgicas, um estudo de revisão apontou que os principais microrganismos infectantes desse tipo de ferida foram as bactérias, seguidas pelos fungos. A análise da literatura publicada sobre o conteúdo evidencia os seguintes microrganismos: S. aureus (39,3\%), E. coli (30,4\%), P. aeruginosa (19,6\%), S. epidermidis (17,8\%), Klesbsiella spp (12,5\%) e Enterobacter spp $(10,7 \%)$ (Santos, et al., 2016). No presente estudo os microrganismos mais prevalentes foram P. mirabilis $(3,2)$, P. aeruginosa $(2,4 \%)$ e E. coli. (1,6\%). Discute-se, assim como nas lesões vasculogênicas, o fato da predominância, nesse estudo, de microrganismos Gram-negativos em lesões nas quais a literatura relata o $S$. aureus como predominante.

Apesar de a maioria das pesquisas apontarem para o $S$. aureus como principal microrganismo colonizador das lesões, devido a sua presença na microbiota da pele, ele não foi o principal encontrado no presente estudo, que apontou a $P$. 
aeruginosa, relacionada com a microbiota hospitalar, em uma maior prevalência. Tal dado pode estar relacionado tanto com o fato de o serviço acompanhar lesões complexas com elevado tempo de cronicidade, quanto pelo fato de atender prioritariamente pacientes portadores de lesão após alta hospitalar, além de desenvolver suas atividades no ambulatório de um hospital.

Esse achado corrobora com os relatados por estudo, que realizou o isolamento, caracterização e identificação de microrganismos em feridas crônicas superficiais e profundas e, apesar de apontarem a $S$. aureus como a principal bactéria da composição do microbiota de ambos os tipos de feridas, o estudo destacou que o aumento na duração do tratamento eleva a presença de bactérias oportunistas, destacando os gêneros Pseudomonas e Enterococcus (Chudobova, et al., 2015). Entretanto, nesse estudo e naqueles resgatados na literatura, fica evidente que a microbiota de lesões complexas, de diferentes etiologias, é predominantemente composta por um microrganismo Gram-negativo e outro Gram-positivo, respectivamente P. aeruginosa e S. aureus.

Para estabelecer e manter uma infecção, a $P$. aeruginosa, microrganismo mais prevalente nesse estudo, expressa mais genes de virulência, com a cronicidade da lesão, a bactéria seleciona clones mais virulentos, gerando fenótipos mais resistentes que persistem por mais tempo com pouca mortalidade associada (Pires, 2018). Por isso, a P. aeruginosa está relacionada a uma alta resistência a antimicrobianos e, sua capacidade de seleção, permite ainda que ela se torne resistente durante o tratamento (Khosravi, et al., 2016).

Por outro lado, o S. aureus colonizador do epitélio humano e microrganismo mais incidente em rupturas da pele, dado não evidenciado nesse estudo, possui como mecanismo de virulência as proteínas de sua parede celular que facilitam sua adesão ao ambiente (Pires, 2018). Em estado crônico, a S. aureus produz metaloproteinases de matriz (MMP) aumentando sua virulência. Destaca-se também a capacidade de suas cepas desenvolverem resistência a múltiplos antimicrobianos, sendo evidenciada as $S$. aureus resistentes a meticilina (MRSA) que são resistentes a todos os antimicrobianos beta-lactâmicos (Oliveira, 2019).

Em relação à resistência antimicrobiana, uma pesquisa que relatou perfil microbiano semelhante ao desse estudo, diferiu ao relatar que a maioria dos microrganismos foram sensíveis à maioria dos antibióticos testados, embora apresentasse resistência a, pelo menos, um antibiótico inibidor de parede celular bacteriana (Borges, Amorim \& Carvalho, 2014). De modo semelhante, um estudo com feridas de pé diabético apontou que apenas parcela dos microrganismos foram multirresistentes aos antimicrobianos testados (Souza, et al., 2016).

Nessa pesquisa, os microrganismos identificados nas lesões avaliadas apresentaram perfil de resistência antimicrobiana acentuado. Esses resultados corroboram com uma tendência de resistência a antibióticos dos microrganismos Gram-negativos relatada nos últimos anos.

Os microrganismos Gram-negativos $P$. aeruginosa e A. baumannii, em um período de quatro anos, apresentaram um aumento significativo da resistência para ceftriaxona, gentamicina, ciprofloxacina, ceftazidima, trimetoprim-sulfametoxazol, cefepima, levofloxacina, piperacilina-tazobactam e amicacina. No entanto, no mesmo período, foi relatado uma diminuição significativa na taxa de resistência da Gram-positiva S. aureus para eritromicina, azitromicina, claritromicina, trimetoprimsulfametoxazol, clindamicina, cefoxitina, norfloxacina, moxifloxacina, gentamicina, tetraciclina, rifampicina, nitrofurantoína, teicoplanina (Mao, et al., 2019).

De modo semelhante, nesse estudo, em relação ao perfil de resistência, destacou-se a bactéria $P$. mirabilis, cuja menor resistência foi observada aos antibióticos trimetoprim-sulfametoxazol e a amicacina. Já em relação à espécie $S$. aureus, embora todas as cepas tenham sido resistentes a penicilina, o presente estudo se assemelha com uma outra pesquisa que mostrou-se sensível a maioria dos antibióticos testados (Pires, 2018).

Devido a constante adaptação dos microrganismos para sobreviverem, destaca-se que a presença de infecção em 
feridas está relacionada às características fisiológicas do portador, bem como aos fatores de virulência e resistência dos microrganismos. Porém a melhora da infecção está mais relacionada a orientações, assistência e ao cuidado de enfermagem especializado (Pires, 2018).

Nesse contexto, existe a necessidade de medidas de controle eficientes no uso de antibióticos (Oliveira \& Oliveira, 2014). Sendo assim, recomenda-se a realização de biópsia de feridas para cultura dos microrganismos a fim de desencorajar a prescrição indiscriminada de antibióticos para evitar a superexposição desnecessária e promoção da resistência antimicrobiana (Tedeschi, et al., 2017).

Quanto ao cuidado de enfermagem, o enfermeiro é o profissional mais habilitado a prescrever a cobertura para o curativo, optando ou não por substâncias bactericidas. Entretanto, escolher a cobertura somente pelas variáveis clínicas pode inferir um erro, visto que alguns microrganismos tem uma alta capacidade de adquirir resistência (Soares, 2019).

Ressalta-se, portanto, a necessidade de se inserir na prática dos cuidados de enfermagem ao portador de ferida a coleta para cultura e o antibiograma, como variáveis de relevância na escolha da terapêutica (Soares, 2019).

\section{Conclusão}

A caracterização da microbiota de feridas complexas e da resistência antimicrobiana dos microrganismos encontrados apontou predominância das bactérias $P$. aeruginosa e $S$. aureus. Em relação à resistência antimicrobiana, todos os microrganismos relatados apresentaram resistência a, pelo menos, um dos antibióticos testados. Estes dados chamam atenção, pois apesar de tratar-se de resultados de exames de pacientes em acompanhamento ambulatorial, as bactérias relatadas são conhecidas pela expressão de fatores de virulência e resistência, bem como presença no ambiente hospitalar.

Os resultados da presente pesquisa embasam o cuidado de enfermagem no tratamento de feridas, uma vez que, apontam a necessidade de maior atenção desses profissionais em relação à colonização e infecção de feridas, bem como do manejo da terapia antimicrobiana. Sugere-se que estudos dessa natureza, são necessários para se compreender os padrões de microbiota de feridas em diferentes contextos e locais. Vislumbra-se, também, a possibilidade da realização de estudos que avaliem a existência de relação entre o resultado da cultura (microrganismo identificado) com a técnica utilizada na coleta do material para o exame de cultura (Swab).

\section{Referências}

Pires, B. M. F. B. (2018) Análise microbiológica e capacidade de formação de biofilme bacteriano em cepas de Pseudomonas aeruginosa e Staphylococcus aureus encontrados em úlceras venosas tratadas com plasma rico em plaquetas (Dissertação de Mestrado). Universidade Federal Fluminense, Niterói, RJ; 2018. https://app.uff.br/riuff/bitstream/1/11809/1/TESE_Bruna\%20Maiara.pdf.

Tedeschi, S., Negosanti, L., Sgarzani, R., Trapani, F., Pignanelli, S., Battilana, M. et al. (2017) Superficial swab versus deep-tissue biopsy for the microbiological diagnosis of local infection in advanced-stage pressure ulcers of spinal-cord-injured patients: a prospective study. Clinical Microbiology and Infection. 23(12), 943-947. 10.1016/j.cmi.2017.04.015.

Haalboom. M, Blokhuis-Arkes. M. H. E., Beuk, R. J., Meerwaldt. R., Klont. R., Schijffelen. M. J., Bowler. P. B., Burnet. M., Sigl. E., \& Van der Pale, J. A. M. (2019) Culture results from wound biopsy versus wound swab: does it matter for the assessment of wound infection? Clinical Microbiology and Infection. 25, 629.e7e629.e12. 10.1016/j.cmi.2018.08.012

Rodrigues, W. C. C., Pinheiro, L. B., Lima, A. T., Battisti, L., Mota, M. A. G., Costa, M. C. et al. (2017) Epidemiological and clinical profile of the burned patients under physiotherapeutic care in the State University of Goiás. Rev Brasileira de Queimaduras 16(2), 9499. http://www.rbqueimaduras.com.br/details/369/pt-BR.

Santos, K. C. B., Ribeiro, G. S. C., Feitosa, A. H. C., Silva, B. R. S., \& Cavalcante, T. B. (2018) Quality of life of hospitalized patients with chronic wounds. Rev. Eletr. Enf. 20 (49). 10.5216/ree.v20.54130.

Santos, W. B., Araujo, M. G. S., Silva, J. C., Bernado, T. H. L., Bastos, M. L. S., \& Verissímo, R. C. S. S. (2016) Surgical wounds infective microbiota: national and international analysis of scientific production. Rev.SOBECC. 21(1), 46-51. 10.5327/Z1414-4425201600010007.

Campos, M. G. C. A., Sousa, A. T. O., Vasconcelos, J. M. B., Lucena, S. A. P., \& Gomes, S. K. A. (2016) Feridas complexas e estomias: aspectos preventivos e manejo clínico. João Pessoa: Ideia. 
Research, Society and Development, v. 10, n. 2, e10110212161, 2021

(CC BY 4.0) | ISSN 2525-3409 | DOI: http://dx.doi.org/10.33448/rsd-v10i2.12161

Souza, G. A. A. D., Garcia, L. M., Rocha, S. S., \& Maciel, A. P. F. (2016) Microbiological profile of skin and soft tissue infections in hospitalized patients from a university hospital. R Epidemiol Control Infec. 6(1), 33-36. 10.17058/reci.v6i1.5901.

Silva, V., Macoleta, A., Silva, A., Flores, D., Aparicio, T., Aburto, I. et al. (2018) Prevalence and susceptibility pattern of bacteria isolated from infected chronic wounds in adult patients. Rev Chil Infectol. 35(2),155-162. 10.4067/s0716-10182018000200155.

Borges, E. L., Amorim, I. P. G., \& Carvalho, D. V. (2014) Características dos Pacientes com Úlcera Venosa Atendidos nas Unidades de Atenção Primária de Nova Lima, Minas Gerais. Estima. 12(1), 31-42. https://www.revistaestima.com.br/index.php/estima/article/view/88.

Oliveira, A. F., \& Oliveira Filho, H. (2014) Microbiological species and antimicrobial resistance profile in patients with diabetic foot infections. J Vasc Bras. 13(4):289-293. 10.1590/1677-5449.0015.

Chudobova, D., Cihalova, K., Guran, R., Dostalova, S., Smerkova, S., Vesely, F. et al. (2015) Influence of microbiome species in hard-to-heal wounds on disease severity and treatment duration. Braz J Infec Dis. 19(6), 604-613. 10.1016/j.bjid.2015.08.013.

Khosravi, A. D., Hovezaivi, H., Mohammadian, A., Farahani, A., \& Jenabi, A. (2016) Genotyping of multidrug-resistant strains of Pseudomonas aeruginosa isolated from burn and wound infections by ERIC-PCR. Acta Cir Bras. 31(3), 206-211. 10.1590/S0102-865020160030000009.

Oliveira, F. P. (2019) Análise microbiológica de feridas tratadas com fator de crescimento epidérmico: estudo de coorte (Tese de Mestrado). Niterói: Universidade Federal Fluminense; 2019. https://app.uff.br/riuff/bitstream/1/11799/1/Fernanda\%20Pessanha\%20de\%20Oliveir a\%20TESE.pdf.

Mao, T., Zhai, H., Duan, Z., \& Yang H. Patterns of Drug-Resistant Bacteria in a General Hospital, China, 2011-2016. Polish J Microb. 2019;68(2):22532. $10.33073 /$ pjm-2019-024.

Soares, R. S. (2019) O perfil bacteriológico e as variáveis relacionadas a ferida neoplásica no paciente em cuidado paliativo (Tese de Mestrado). Niterói: Universidade Federal Fluminense. https://app.uff.br/riuff/bitstream/1/9596/1/Raquel\%20de\%20Souza\%20Soares.pdf. 